\title{
ASYMPTOTIC SELF-SIMILAR BLOW-UP FOR A MODEL OF AGGREGATION
}

\author{
IGNACIO GUERRA \\ Centre for Mathematical Modeling, Universidad de Chile, FCFM \\ Casilla 170 Correo 3, Santiago, Chile \\ E-mail: iguerra@dim.uchile.cl
}

\begin{abstract}
In this article we consider a system of equations that describes a class of massconserving aggregation phenomena, including gravitational collapse and bacterial chemotaxis. In spatial dimensions strictly larger than two, and under the assumptions of radial symmetry, it is known that this system has at least two stable mechanisms of singularity formation (see e.g. M. P. Brenner et al. 1999, Nonlinearity 12, 1071-1098); one type is self-similar, and may be viewed as a trade-off between diffusion and attraction, while in the other type the attraction prevails over the diffusion and a non-self-similar shock wave results. Our main result identifies a class of initial data for which the blow-up behaviour is of the former, self-similar type. The blow-up profile is characterized as belonging to a subset of stationary solutions of the associated ordinary differential equation. We compare these results with blow-up behaviour of related models of aggregation.
\end{abstract}

1. Introduction. We consider the parabolic-elliptic system

$$
\begin{array}{cl}
n_{t}=\operatorname{div}\{\Theta \nabla n+n \nabla \phi\} & \text { in } \Omega \times \mathbb{R}^{+}, \\
\Delta \phi=n & \text { in } \Omega \times \mathbb{R}^{+}, \\
0=(\Theta \nabla n+n \nabla \phi) \cdot \vec{\nu} & \text { on } \partial \Omega \times \mathbb{R}^{+}, \\
\phi=0 & \text { in } \partial \Omega \times \mathbb{R}^{+}, \\
n(x, 0)=n_{0}(x) & \text { in } \Omega,
\end{array}
$$

where $\Omega=B_{1}(0)=\left\{x \in \mathbb{R}^{d}:|x| \leq 1\right\}, d>2$, and $\vec{\nu}$ is the outer normal vector from the boundary $\partial \Omega$. Here $\Theta>0$ is a constant parameter. The initial condition $n_{0}$ is chosen in

2000 Mathematics Subject Classification: 35Q, 35K60, 35B40, 82C21.

Key words and phrases: aggregation of particles models, nonlinear nonlocal parabolic system, finite time blow-up of solutions.

The paper is in final form and no version of it will be published elsewhere. 
$L^{2}(\Omega)$, radially symmetric and such that

$$
\int_{\Omega} n_{0} d x=1, \quad \text { and } \quad n_{0}(x) \geq 0 \quad \text { in } \quad \Omega .
$$

Equations (1)-(6) define a problem for the unknown mass density $n$ and potential $\phi$. We observe that since $\phi$ is known in terms of $n$ by the boundary value problem (2) and (4), we denote a solution of (1)-(6) simply by $n$. By the no-flux condition (3), mass is preserved. In fact, condition (6) gives

$$
\int_{\Omega} n(x, t) d x=\int_{\Omega} n_{0}(x) d x=1 .
$$

Problem (1)-(6) is a model for the evolution of a cluster of particles under gravitational interaction and Brownian motion, see [5] and references therein. Suppose that we have a cluster of particles with: total mass $M a$, particle mass $m a$, radius $R a$, and temperature $\vartheta$. The parameter $\Theta$ is a rescaled temperature, incorporating masses in the system:

$$
\Theta=\frac{\kappa \cdot \vartheta \cdot R a^{d-2}}{G \cdot M a \cdot m a} .
$$

Here $\kappa$ and $G$ are the Boltzmann and gravitational constants. Since temperature is constant, problem (1)-(6) is called the isothermal model [17]. This model also appears in the study of evolution of polytropic stars, by considering the evolution of self-interacting clusters of particles under frictional and fluctuating forces [38].

Problem (1)-(6) also arises in the study of the motion of bacteria by chemotaxis as a simplification (see [29]) of the Keller-Segel model [30]. We refer to [37, 2, 9] for recent results. In this case, the variables $n$ and $\phi$ represent the density of bacteria and the concentration of the chemo-attractant.

In this paper will view the problem (1)-(6) as an evolution equation in $n$, since by equations $(2-3)$ the function $\phi$ is readily recovered from the solution $n$. It is known [6] that problem (1)-(6) has a unique local solution if $n_{0} \in L^{2}(\Omega)$, which satisfies $n \in$ $L^{\infty}(\Omega \times(\epsilon, \tilde{T}))$ for some $\tilde{T}>0$ and for every $\epsilon>0$. Now, since $\Omega=B_{1}(0)$ and $n_{0}$ is radially symmetric, by uniqueness this local solution is radially symmetric. For that reason, we restrict ourselves to the analysis of radial solutions and write $n(r, t):=n(x, t)$ with $r=|x| \in[0,1]$.

Since we are interested in the question when and how the isothermal model generate singularities, we define:

$$
T^{*}=\sup \left\{\tau>0 \mid \text { Problem (1)-(6) has a solution } n \in L^{\infty}(\Omega \times(\epsilon, \tau])\right\} .
$$

If $T^{*}<\infty$, then we say that blow-up occurs for (1)-(6), more precisely, one finds

$$
\lim _{t \rightarrow T^{*}} \sup _{[0,1]} n(r, t)=\infty \text {. }
$$

There are various conditions in the literature which ensure $T^{*}<\infty[3,4,7,6]$. For instance, in [4] it was found for the radially symmetric case that $T^{*}<\infty$ holds for any bounded initial condition and $\Theta<1 /\left(2 d^{2} \chi_{d}\right)$. Here $\chi_{d}$ is the volume of the unit ball in $\mathbb{R}^{d}$. However, from the proof of these results we cannot infer how the blow-up occurs. The aim of this paper is to characterize the asymptotic behaviour near blow-up of the 
solution $n(r, t)$ of problem (1)-(6). Let $n_{0}=n_{0}(r)$ be such that

$$
\chi_{d} r^{d} n_{0}(r) \leq\left\|n_{0}\right\|_{L^{1}\left(B_{r}(0)\right)} \text { for } r \in(0,1),
$$

$$
\Theta\left(n_{0}\right)_{r}+n_{0}\left(\phi_{0}\right)_{r} \geq 0, \quad\left(r^{d}\left(\phi_{0}\right)_{r}\right)_{r}=r^{d} n_{0}, \quad \text { in }(0,1) \text { and } \phi_{0}(1)=0 .
$$

Suppose also that $\Theta \leq 1 /\left(4 d \chi_{d}\right)$ and the two functions

$$
\left\|n_{0}\right\|_{L^{1}\left(B_{r}(0)\right)} \text { and } \frac{4 \Theta r^{d}}{2(d-2) \Theta T^{*}+r^{2}} \text { intersect exactly once in }[0,1] \text {. }
$$

From the bound on temperature it follows [4] that the solution $n=n(r, t)$ of (1)-(6) blows up at finite time $T^{*}>0$ and at the point $r=0$, and so we can write (11). Our main result (Theorem 2.1) shows that if (9), (10), and (11) hold, then $n$ satisfies

$$
n(0, t) \leq \frac{2 d}{(d-2)}\left(T^{*}-t\right)^{-1} \quad \text { for } \quad t \in\left(0, T^{*}\right),
$$

and moreover has a structure near blow-up given by

$$
n_{*}(r, t)=\left(T^{*}-t\right)^{-1} \Psi\left(\frac{r}{\sqrt{\Theta\left(T^{*}-t\right)}}\right),
$$

where the function $\Psi$ belongs to a class of solutions of a steady-state problem; a class that includes the functions

$$
\Psi_{1}(\eta):=(d-2) \frac{\left(2 d+\eta^{2}\right)}{\left(d-2+\frac{1}{2} \eta^{2}\right)^{2}} \quad \text { and } \quad \Psi^{*}(\eta):=1 \quad \text { for } \quad \eta>0 .
$$

In particular, if $n_{0} \equiv 1 / \chi_{d}$ and $\Theta \leq 1 /\left(4 d \chi_{d}\right)$ the above conclusion also holds (Corollary 2.2). If we relax assumption (11) but assume instead that $n$ satisfies the growth condition

$$
n(0, t) \leq M\left(T^{*}-t\right)^{-1} \quad \text { for } \quad t \in\left(0, T^{*}\right),
$$

for some constant $M>0$, then $n$ has the same structure of blow-up given above. (Theorem 2.3). The hypotheses on the initial data (9), (10), and (11) are more natural in the context of a transformed problem we introduce in the next section. Note however that $\left(n_{0}\right)_{r} \leq 0$ in $[0,1]$ implies assumption (9).

For $d=3$, the problem of blow-up was first studied by Herrero et al. [27, 28] using careful matched asymptotic expansions. Later Brenner et al. [11] studied the problem for $2<d<10$. They use numerical analysis to describe solutions and also prove existence and linear stability of self-similar profiles. Note however that no proof of convergence or characterization of blow-up in terms of initial data were given in these references. The principal types of blow-up described in [27, 28, 11] are:

(a) A solution $n(r, t)$ consists of an imploding smoothed-out shock wave which moves towards the origin. As $t \rightarrow T^{*}$, the bulk of such a wave is concentrated at distances $O\left(\left(T^{*}-t\right)^{1 / d}\right)$ from the origin, has a width $O\left(\left(T^{*}-t\right)^{(d-1) / d}\right)$, and at its peak it reaches a height of order $O\left(\left(T^{*}-t\right)^{-2(d-1) / d}\right)$. This type of blow-up has the property of concentration of mass at the origin at the blow-up time, i.e.

$$
\lim _{r \rightarrow 0}\left[\lim _{t \rightarrow T^{*}} \int_{0}^{r} n(y, t) y^{d-1} d y\right]=C>0 .
$$


This situation is known as gravitational or chemotactic collapse and is depicted in Figure 1 (left). The class of initial data considered in this paper rules out this possibility.

(b) A solution $n(r, t)$ has a self-similar blow-up of the form

$$
\left(T^{*}-t\right) n\left(\eta \sqrt{\left(T^{*}-t\right) \Theta}, t\right) \sim \Psi_{1}(\eta) \quad \text { as } \quad t \rightarrow T^{*} .
$$

Note that this implies that $n$ satisfies (12) with $C=0$. Therefore no concentration of mass at the origin occurs at the blow-up time. This blow-up behaviour is depicted in Figure 1 (right) and is the type of behaviour that we obtain in the results of this paper.

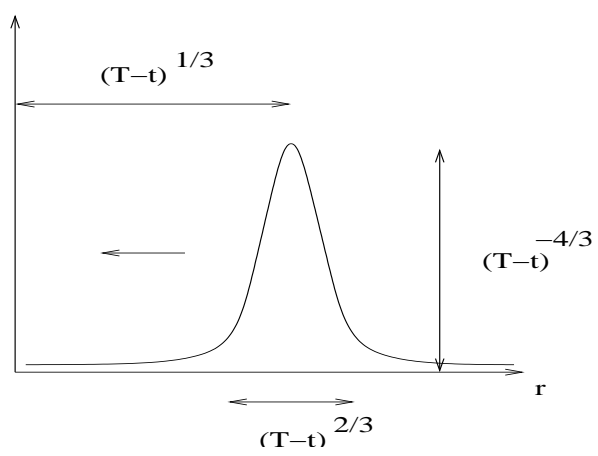

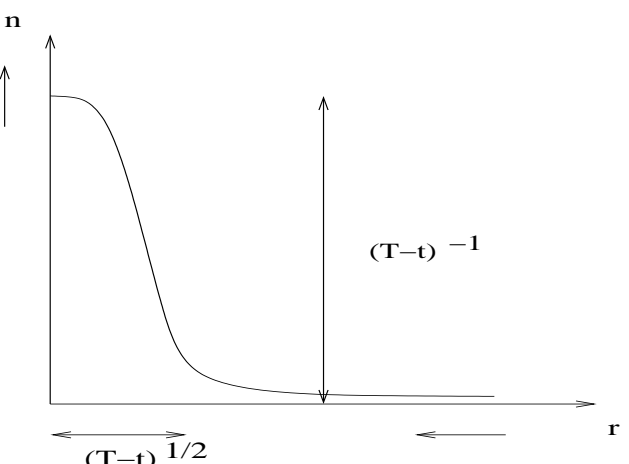

Fig. 1. The profile $n(r, t)$ for blow-up with (left) and without (right) concentration of mass, with $T=T^{*}$

The article is organized as follows. In Section 2, we reformulate the problem in terms of a new variable, transforming the system (1)-(6) into a single PDE. We state our results in this new formulation. In Section 3, we discuss non-self-similar blow-up patterns related to case (a). Sections 4 and 5 are devoted to the proof of Theorem 2.3. The idea is to change variables and put the system in self-similar form, so that blow-up profiles can be seen as stationary solutions. We prove stabilization towards blow-up profiles and moreover we show that they belong to a subset of solutions of the stationary problem. In Section 6 we prove intersection comparison results and in Section 7 we use these results to show that the stabilization gives the convergence to a single function. This fact together with a comparison with a singular solution proves Theorem 2.3. In addition, using comparison with bounded solutions, we obtain Theorem 2.1 and Corollary 2.2. In Section 8, we study linear stability of the blow-up profiles. Finally, in the last section, we compare our results with blow-up results of related models.

2. Main results. For radial solutions, the average density function $b(r, t)[11]$ is defined by

$$
b(r, t):=\frac{d \chi_{d}}{r^{d}} \int_{0}^{r} n(y, t) y^{d-1} d y,
$$


This variable turns out to be convenient when analyzing this system. Note that it has the same scale invariance as $n(r, t)$, but solutions are smoother when expressed in terms of $b$. For example, if for some fixed $t>0$ the density $n(r, t)$ is a delta function at the origin with unit mass, then $b(r, t)=r^{-d}$. Let $D=(0,1)$ and set $D_{T}=D \times(0, T)$ for some $T>0$. Transformation (13) puts system (1)-(6) in the form

$$
\begin{gathered}
b_{t}=\chi_{d} \Theta\left(b_{r r}+\frac{d+1}{r} b_{r}\right)+\frac{1}{d} r b b_{r}+b^{2} \text { in } D_{T}, \\
b_{r}(0, t)=0, \quad b(1, t)=1, \text { for } t \in[0, T), \\
b(0, r)=b_{0}(r) \text { for } r \in D .
\end{gathered}
$$

Here we have redefined $t:=\frac{1}{\chi_{d}} t$. Regarding the initial condition, we assume

$$
b_{0} \in C^{2}(\bar{D}), \quad \text { and } \quad \frac{r}{d}\left(b_{0}\right)_{r}+b_{0} \geq 0 \text { for } r \in D,
$$

where the second condition is equivalent to $n_{0} \geq 0$ in $D$. Note that the conservation of the mass $(7)$ is represented by $b(1, t)=1$ for $t \in[0, T)$. As was done for problem (1)-(6) we define $T>0$ to be the maximal time of existence for the average density $b(r, t)$. If $T^{*}<\infty$ in (8), then

$$
\lim _{t \rightarrow T} \sup _{[0,1]} b(r, t)=\infty,
$$

where $T=T^{*} / \chi_{d}$. Using (13), we deduce $b(r, t) \leq 1 / r^{d}$ for $r \in \bar{D}, t>0$; this implies single point blow-up for $b(r, t)$ at the point $r=0$. To characterize the asymptotic behaviour near blow-up of the solution $b(r, t)$ of problem (14)-(17), we study the solutions of the associated boundary-value problem

$$
\left\{\begin{array}{l}
\varphi_{\eta \eta}+\frac{d+1}{\eta} \varphi_{\eta}+\frac{1}{d} \eta \varphi \varphi_{\eta}-\frac{1}{2} \eta \varphi_{\eta}+\varphi^{2}-\varphi=0, \quad \text { for } \quad \eta>0 \\
\varphi(0) \geq 1 \quad \varphi_{\eta}(0)=0
\end{array}\right.
$$

If $b$ is a solution of (14)-(17) which blows up at time $T>0$ and at the point $r=0$, then we will show that it has the asymptotic form given by

$$
b_{*}(r, t)=(T-t)^{-1} \varphi\left(\frac{r}{\sqrt{\chi_{d} \Theta(T-t)}}\right) .
$$

Equation (18) has multiple solutions for $2<d<10[28,11]$. We classify them by counting the number of times they cross the singular solution $\varphi_{S}(\eta):=2 d / \eta^{2}$. For that purpose, we introduce the set

$$
\mathcal{S}_{k}=\left\{\varphi: \varphi \text { is a solution of (18) having } k \text { intersections with } \varphi_{S}\right\} .
$$

We shall see that $\mathcal{S}_{1}$ is the relevant subset of solutions of (18) for the characterization of blow-up. Numerical evidence [11] suggests that $\mathcal{S}_{1}$ contains only two elements:

$$
\varphi^{*}(\eta)=1 \quad \text { and } \quad \varphi_{1}(\eta):=\frac{2 d}{\left(d-2+\frac{\eta^{2}}{2}\right)} \quad \text { for } \quad \eta \geq 0 .
$$

For the initial condition, we assume

$$
\left(b_{0}\right)_{r} \leq 0 \text { for } r \in D,
$$


and

$$
\chi_{d} \Theta\left(\left(b_{0}\right)_{r r}+\frac{d+1}{r}\left(b_{0}\right)_{r}\right)+\frac{1}{d} r b_{0}\left(b_{0}\right)_{r}+b_{0}^{2} \geq 0 \quad \text { for } \quad r \in D .
$$

We will show that this implies $b_{r} \leq 0$ in $D_{T}$ and $b_{t} \geq 0$ in $D_{T}$. In terms of $n_{0}$ assumption (20) becomes (9) and assumption (21) becomes (10).

TheOREM 2.1. Let $d>2$ and $b_{0}$ satisfy (20) and (21). Let $b(r, t)$ be the corresponding solution of problem (14)-(17) that blows up at $r=0$ and at $t=T$. If

$$
\Theta \leq \Theta_{1}:=1 /\left(4 d \chi_{d}\right) \quad \text { and } \quad b_{0}(r) \text { intersects } T^{-1} \varphi_{1}\left(r / \sqrt{\chi_{d} \Theta T}\right) \text { once }
$$

then

$$
b(0, t) \leq M_{1}(T-t)^{-1} \quad \text { for } \quad t \in(0, T)
$$

with $M_{1}:=2 d /(d-2), T<M_{1} / b_{0}(0)$, and there exists $\varphi \in S_{1}$ such that

$$
\lim _{t \rightarrow T}(T-t) b\left(\eta \sqrt{\chi_{d} \Theta(T-t)}\right)=\varphi(\eta)
$$

uniformly on compact sets $|\eta| \leq C$ for every $C>0$.

We remark that there exists a family of $b_{0}$ satisfying the conditions (17), (20), and (21), given by $b_{0}(r)=K_{1}+K_{2} /\left(r^{d}+K_{3}\right)$ with positive constants $K_{i}$ that satisfy $K_{1}+$ $K_{2} /\left(1+K_{3}\right)=1$ and $\Theta<K_{2} / 2 d^{2} \chi_{d}$. Conditions (17), (20), and (21) are also satisfied for $b_{0} \equiv 1$. Note that condition (22) of Theorem 2.1 can be generalized by changing $\varphi_{1}$ for other solution $\varphi$ of (18). Since these solutions are only known numerically, the counterpart of $M_{1}$ and $\Theta_{1}$ cannot be given explicitly. The next corollary applies this result to $b_{0} \equiv 1$. Corollary 2.2. Let $d>2, b_{0} \equiv 1$ and $\Theta<\Theta_{1}$. Then $b(r, t)$, the corresponding solution of problem (14)-(16), blows up at $r=0$ and at some time $t=T<M_{1}$; moreover (23) holds and there exists $\varphi \in S_{1}$ such that the convergence (24) holds.

Numerical simulations [11] suggest that for an open set of initial data the convergence in (24) holds for $\varphi=\varphi_{1}$. This self-similar behaviour is depicted in Figure 1 (right), by replacing $n(r, t)$ by $b(r, t)$. In Section 8 we show that $\varphi_{1}$ is linearly stable (using the result in $[11])$ and also that $\varphi^{*}$ is linearly unstable.

For more general initial data we have the following result.

Theorem 2.3. Let $d>2$ and let $b_{0}$ satisfy (20) and (21). Assume that $b(r, t)$, the corresponding solution of problem (14)-(17), blows up at $r=0$ and at $t=T$. If b satisfies the growth condition

$$
b(0, t) \leq M(T-t)^{-1} \quad \text { for } \quad t \in(0, T)
$$

with $M>0$, then there exists $\varphi \in S_{1}$ such that the convergence (24) holds.

For the proof of these theorems we introduce self-similarity in (14)-(17). Using scale invariance, we set:

$$
\tau=\log \left(\frac{T}{T-t}\right) \quad \text { and } \quad \eta=\frac{r}{\left(\chi_{d} \Theta(T-t)\right)^{1 / 2}} ;
$$

and for the unknown $b$ we define

$$
B(\eta, \tau)=(T-t) b(r, t) .
$$


The rectangle $D_{T}$ transforms into

$$
\Pi=\{(\eta, \tau) \mid \tau>0,0<\eta<\ell(\tau)\} \quad \text { where } \quad \ell(\tau):=\left(\chi_{d} \Theta T\right)^{-1 / 2} e^{\tau / 2} .
$$

The initial-boundary problem (14)-(17) now becomes

$$
\begin{array}{r}
B_{\tau}+B+\frac{1}{2} \eta B_{\eta}=B_{\eta \eta}+\frac{d+1}{\eta} B_{\eta}+\frac{1}{d} \eta B B_{\eta}+B^{2} \text { in } \Pi, \\
B_{\eta}(0, \tau)=0, \quad B(\ell(\tau), \tau)=e^{-\tau} T \text { for } \tau \in \mathbb{R}^{+}, \\
B(\eta, 0)=B_{0}(\eta):=T b_{0}\left(\eta\left(\chi_{d} \Theta T\right)^{1 / 2}\right) \text { for } \eta \in \Pi(0),
\end{array}
$$

where $\Pi(0)=(0, \ell(0))$. Note that a solution of $(18)$ is a time-independent solution of $(29)$ (31). Therefore the study of the blow-up behaviour of $b(r, t)$ is reduced to the analysis the large time behaviour of solutions $B(\eta, \tau)$ of $(29)-(31)$, and in particular stabilization towards solutions $\varphi$ of (18). The proof of Theorem 2.3 consists of two parts. In Section 5 , we first prove that $\omega \subset \mathcal{S}_{1}$, where

$$
\begin{aligned}
\omega= & \left\{\phi \in L^{\infty}\left(\mathbb{R}^{+}\right) \mid \exists \tau_{j} \rightarrow \infty\right. \text { such that } \\
& \left.B\left(\cdot, \tau_{j}\right) \rightarrow \phi(\cdot) \text { as } \tau_{j} \rightarrow \infty \text { uniformly on compact subsets of } \mathbb{R}^{+}\right\}
\end{aligned}
$$

is the $\omega$-limit set we introduce for (29)-(31). The proof uses the observation that equation (29), without the convection term $\frac{1}{d} \eta B B_{\eta}$, is the backward self-similar equation for the parabolic semilinear equation

$$
\bar{b}_{t}=\Delta_{N} \bar{b}+\bar{b}^{2}
$$

where $\Delta_{N}$ denotes the Laplacian in $\mathbb{R}^{N}$ and $N=d+2[21,22]$. We use the methods for the analysis of this self-similar equation to prove Theorem 2.3. However, due to the presence of the convection term, a different Lyapunov functional is necessary. This functional is constructed using the method of Zelenyak [39], which yields a Lyapunov functional in implicit form. In Section 6, we use intersection comparison arguments based on the ideas of Matano [31] to prove that the $\omega$-limit set (32) is a singleton. With a result on intersection with $\varphi_{S}$ this completes the proof of Theorem 2.3.

Note that Theorem 2.3 is similar to a result for the supercritical case $(N>6)$ for equation (33), where two different kinds of self-similar blow-up behaviour may coexist [32].

Finally to obtain Theorem 2.1 and Corollary 2.2, we use Theorem 2.3 and comparison ideas from Samarskii et al. [34, Chapter IV].

3. Discussion on non self-similar blow-up patterns. In this section we discuss a family of blow-up patterns which appears when we refine the asymptotic expansion for the profile $\varphi=\varphi^{*} \equiv 1$. This situation is closely related to the blow-up behaviour of (33) with $N<6$. If a solution $\bar{b}$ of (33) with $N<6$ blows up at $x=0$ and $t=T$, then

$$
\lim _{t \rightarrow T}(T-t) \bar{b}(\eta \sqrt{T-t}, t)=1
$$

uniformly on compact sets $|\eta|<C$ for arbitrary $C>0[21,22]$. Moreover it have been shown (see for instance $[33,36]$ ) that a refined description of blow-up gives the existence 
of two possible types of behaviour: either

$$
\lim _{t \rightarrow T}(T-t) \bar{b}(\eta \sqrt{(T-t)|\log (T-t)|}, t)=\bar{\varphi}_{1}(\eta)
$$

uniformly on compact sets $|\eta|<C$, with $C>0$ arbitrary; or

$$
\lim _{t \rightarrow T}(T-t) \bar{b}\left(\eta(T-t)^{1 / 2 m}, t\right)=\bar{\varphi}_{m}(\eta) \quad \text { for some } \quad m \geq 2,
$$

uniformly on compact sets $|\eta|<C$, with $C>0$ arbitrary. Here the family $\left\{\bar{\varphi}_{i}\right\}_{i \geq 1}$ is known explicitly. For problem (14)-(17), it was shown [28] for $d=3$ that there exists a refined asymptotics for $\varphi^{*} \equiv 1$. Extending the argument to all $d>2$, these asymptotics suggest a convergence given by either

$$
\lim _{t \rightarrow T}(T-t) b\left(\eta \sqrt{(T-t)|\log (T-t)|^{(d-2) / d}}, t\right)=\tilde{\varphi}_{1}(\eta)
$$

in the case $d=3,4$ only, or

$$
\lim _{t \rightarrow T}(T-t) b\left(\eta(T-t)^{1 / d+1 /(2(m+[d /(d-2)]-1))}, t\right)=\tilde{\varphi}_{m}(\eta)
$$

for some $m \geq 2$, where $[x]$ denotes the greatest integer $\leq x$. An implicit formula for the family $\left\{\tilde{\varphi}_{m}\right\}_{m \geq 1}$ is given in [11, equation (43)]. The type of convergence in $\eta$ towards these profiles is an open problem. In (37), we can take formally the limit $m \rightarrow \infty$ and find a non-trivial scaling,

$$
\lim _{t \rightarrow T}(T-t) b\left(\eta(T-t)^{1 / d}, t\right)=\tilde{\varphi}_{\infty}(\eta) .
$$

Note that this limit cannot be taken for the semilinear equation where (35) holds. The convergence (38) represents the convection-dominant behaviour of (14)-(17), which in terms of the density $n=n(r, t)$ describes an imploding wave moving towards the origin, as shown in Figure 1 (left). The function $\tilde{\varphi}_{\infty}$ is discontinuous (cf. [27, (3.16)]),

$$
\tilde{\varphi}_{\infty}(\eta)=\left\{\begin{array}{lll}
\frac{2 C^{d}}{\eta^{d}} & \text { for } & \eta>C \\
0 & \text { for } & \eta<C,
\end{array}\right.
$$

where $2 C^{d}$ is the mass accumulated in the origin, which can be chosen arbitrarily. In [27] this type of blow-up was studied using matched asymptotic expansions. There it was suggested that this behaviour is stable and moreover it was expected that there exist initial data such that (38) holds uniformly in $\eta$ on compact subsets away from the shock. A result of this type was proved in [18, Theorem 3] for a related equation.

\section{Preliminaries}

4.1. Estimates. In this section we develop some estimates for problem (14)-(16), which in turn will imply bounds for the self-similar problem (29)-(31).

LEMMA 4.1 ([24]). 1. If $b_{0}$ satisfies (17) then

$$
\frac{r}{d} b_{r}+b \geq 0 \quad \text { in } \quad D_{T}
$$

2. if $b_{0}$ satisfies (20) then

$$
b_{r}(r, t)<0 \quad \text { in } \quad D_{T} \text {; }
$$


3. if $b_{0}$ satisfies (20) and assuming that blow-up occurs at time $T>0$, then

$$
b(0, t) \geq(T-t)^{-1} \quad \text { for } \quad t \in[0, T) ;
$$

4. if $b_{0}$ satisfies $(21)$ then $b_{t} \geq 0$ for all $t \in(0, T)$;

5. if $b_{0}$ satisfies (20) and (21), then

$$
\chi_{d} \Theta b_{r}^{2}(r, t) \leq \frac{2}{3} b(0, t)^{3} \quad \text { for } \quad(r, t) \in D_{T} .
$$

To conclude, we translate the properties of solutions derived above into estimates for problem (29)-(31). From hypothesis (25) and noting that $b \geq 1$ and $b_{r} \leq 0$ in $D_{T}$, we have the a priori bound

$$
0 \leq B(\eta, \tau) \leq M \quad \text { for } \quad(\eta, \tau) \in \Pi
$$

Combining this with (42) and (40), we obtain

$$
0 \leq-B_{\eta}(\eta, \tau) \leq \bar{M} \text { for }(\eta, \tau) \in \Pi,
$$

where $\bar{M}$ depends on $M$. Finally from (41), we get

$$
1 \leq B(0, \tau) \quad \text { for } \quad \tau \in(0, \ell(\tau))
$$

4.2. The steady state equation (18). We begin recalling problem (18):

$$
\begin{aligned}
\varphi_{\eta \eta}+\frac{d+1}{\eta} \varphi_{\eta}+\frac{1}{d} \eta \varphi \varphi_{\eta}-\frac{1}{2} \eta \varphi_{\eta}+\varphi^{2}-\varphi & =0 \quad \text { for } \quad \eta>0 \\
\varphi(0) & \geq 1, \quad \varphi_{\eta}(0)=0 .
\end{aligned}
$$

Condition (47) is required, since $B(0, \tau) \geq 1$ for all $\tau \geq 0$. Equation (46) has three special solutions:

$$
\varphi_{S}(\eta)=\frac{2 d}{\eta^{2}}, \quad \varphi^{*}(\eta)=1, \quad \text { and } \quad \varphi_{*}(\eta)=0 \quad \text { for } \quad \eta>0
$$

Note that $\varphi_{S}$ satisfies

$$
\varphi_{S}+\frac{1}{2} \eta\left(\varphi_{S}\right)_{\eta}=0 \quad \text { and } \quad 0=\left(\varphi_{S}\right)_{\eta \eta}+\frac{d+1}{\eta}\left(\varphi_{S}\right)_{\eta}+\frac{1}{d} \eta \varphi_{S}\left(\varphi_{S}\right)_{\eta}+\left(\varphi_{S}\right)^{2} .
$$

For bounded non-constant solutions we have the following theorem $[11,28]$.

ThEOREM 4.2. Let $2<d<10$. There exists a countable set of solutions $\left\{\varphi_{k}\right\}_{k \in \mathbb{N}}$ of (46)-(47) such that $\varphi_{k}(0)>1$ and $\varphi_{k}(0) \rightarrow \infty$ as $k \rightarrow \infty$, Moreover $\varphi_{k}$ intersects the singular solution $\varphi_{S} k$ times and has the asymptotic behaviour $\varphi_{k}(\eta) \eta^{2}=\operatorname{Const}(k)>0$.

The proof is based on the equation for $G(\eta):=\eta^{2} \varphi(\eta)$,

$$
\begin{gathered}
G_{\eta \eta}+\left(\frac{(d-3)}{\eta}+\frac{1}{d} \frac{G}{\eta}-\frac{1}{2} \eta\right) G_{\eta}+\frac{2(d-2) G}{\eta^{2}}\left(\frac{G}{2 d}-1\right)=0, \\
\lim _{\eta \downarrow 0} \frac{G(\eta)}{\eta^{2}}<\infty, \quad \lim _{\eta \rightarrow \infty} \eta G_{\eta}(\eta)=0 .
\end{gathered}
$$

Note that $\varphi_{S}$ corresponds to $G(\eta) \equiv 2 d$.

It was formally argued in [11] that for each integer $k \geq 2$ and $2<d<10$ the set

$$
\mathcal{S}_{k}=\left\{\varphi: \varphi \text { solution of }(46)-(47) \text { with } k \text { intersections with } \varphi_{S}\right\}
$$


is a singleton and that for $d>2$ the set $\mathcal{S}_{1}$ contains only two elements. More precisely, $\mathcal{S}_{1}$ consists of the functions $\varphi^{*}$ and $\varphi_{1}$ in (19). If we relax condition (47) to $\varphi(0)>0$, we can find at least one other solution in $\mathcal{S}_{1}$. For $d=3$, Brenner et al. found numerically a solution $\varphi_{1}^{*}$ of $(46)$ such that $\varphi_{1}^{*}(0)<1$ and $\left(\varphi_{1}^{*}\right)_{\eta}(0)=0$, which intersects $\varphi_{S}$ once [11, Figure 14].

5. Convergence. In this section we prove the following convergence theorem.

TheOREM 5.1. Let conditions (20) and (21) hold. Let $B(\eta, \tau)$ be a uniformly bounded global solution of (29)-(31). Then for every sequence $\tau_{n} \rightarrow \infty$ there exists a subsequence $\tau_{n}^{\prime}$ such that $B\left(\eta, \tau_{n}^{\prime}\right)$ converges to a solution $\varphi$ of $(46)-(47)$. The convergence is uniform on every compact subset of $[0, \infty)$.

Proof. Define $B^{\sigma}(\eta, \tau):=B(\eta, \sigma+\tau)$. We will first show that for any unbounded sequence $\left\{n_{j}\right\}$ there exists a subsequence (renamed $\left\{n_{j}\right\}$ ) such that $B^{n_{j}}$ converges to a solution $\varphi$ of (46)-(47) uniformly in compact subsets of $\mathbb{R}^{+} \times \mathbb{R}$. Without loss of generality we assume that the sequence $\left\{n_{j}\right\}$ is increasing.

Let $N \in \mathbb{N}$. We take $i$ large enough such that the rectangle $\mathcal{Q}_{2 N}=\left\{(\eta, \tau) \in \mathbb{R}^{2}: 0 \leq\right.$ $\eta \leq 2 N,|\tau| \leq 2 N\}$ lies in the domain of $B^{n_{i}}$. The function $\tilde{B}(\xi, \tau)=B^{n_{i}}(|\xi|, \tau)$ is a solution of

$$
\tilde{B}_{\tau}=\Delta_{d+2} \tilde{B}-\frac{1}{2} \xi \cdot \nabla \tilde{B}+\frac{1}{d}(\xi \cdot \nabla \tilde{B}) \tilde{B}+\tilde{B}^{2}-\tilde{B}
$$

on the cylinder given by

$$
\Gamma_{2 N}=\left\{(\xi, \tau): \mathbb{R}^{d+2} \times \mathbb{R}:|\xi| \leq 2 N,|\tau| \leq 2 N\right\},
$$

and $|\tilde{B}(\xi, \tau)|$ is uniformly bounded in $\Gamma_{2 N}$ by $(43)$.

By Schauder's interior estimates all partial derivatives of $\tilde{B}$ can be uniformly bounded on the subcylinder $\Gamma_{N} \subset \Gamma_{2 N}$. Consequently $B^{n_{i}}, B_{\tau}^{n_{i}}, B_{\eta}^{n_{i}}$, and $B_{\eta \eta}^{n_{i}}$ are uniformly Lipschitz on $\mathcal{Q}_{N} \subset \mathcal{Q}_{2 N}$. By Arzela-Ascoli, there is a subsequence $\left\{n_{j}\right\}_{1}^{\infty}$ and a function $\bar{B}$ such that $B^{n_{i}}, B_{\tau}^{n_{i}}, B_{\eta}^{n_{i}}$, and $B_{\eta \eta}^{n_{i}}$ converge to $\bar{B}, \bar{B}_{\tau}, \bar{B}_{\eta}$, and $\bar{B}_{\eta \eta}$, uniformly on $\mathcal{Q}_{N}$.

Repeating the construction for all $N$ and taking a diagonal subsequence, we can conclude that

$$
B^{n_{j}} \rightarrow \bar{B}, \quad B_{\tau}^{n_{j}} \rightarrow \bar{B}_{\tau}, \quad B_{\eta}^{n_{j}} \rightarrow \bar{B}_{\eta}, \quad \text { and } \quad B_{\eta \eta}^{n_{j}} \rightarrow \bar{B}_{\eta \eta}
$$

uniformly in every compact subset in $\mathbb{R}^{+} \times \mathbb{R}$. Clearly $\bar{B}$ satisfies (29) and estimates (43) and (44). Finally, it remains to prove that $\bar{B}$ is independent of $\tau$. This implies that $\bar{B}$ is a solution of $(18)$, since $B(0, \tau) \geq 1$ for all $\tau>0$, and the result follows.

Claim. The function $\bar{B}$ is independent of $\tau$.

To prove this we construct a non-explicit Lyapunov functional in the spirit of Galaktionov [20] and Zelenyak [39].

Non-explicit Lyapunov functional. We seek a Lyapunov function of the form

$$
E(\tau)=\int_{0}^{\ell(\tau)} \Phi\left(\eta, B(\eta, \tau), B_{\eta}(\eta, \tau)\right) d \eta
$$


where $\ell(\tau)=\left(\chi_{d} \Theta T\right)^{-1 / 2} e^{\tau / 2}$ and $\Phi=\Phi(\eta, v, w)$ is a function to be determined. In Appendix A we show that such a Lyapunov function exists; more precisely, we show that a function $\rho=\rho(\eta, v, w)$ exists such that

$$
\begin{aligned}
\frac{d}{d \tau} E(\tau)= & -\int_{0}^{\ell(\tau)} \rho\left(\eta, B(\eta, \tau), B_{\eta}(\eta, \tau)\right)\left(B_{\tau}\right)^{2}(\eta, \tau) d \eta \\
& +\left.\Phi_{w} B_{\tau}\right|_{0} ^{\ell(\tau)}+\frac{1}{2} \ell(\tau) \Phi\left(\ell(\tau), B(\ell(\tau), \tau), B_{\eta}(\ell(\tau), \tau)\right) .
\end{aligned}
$$

To identify the relevant domain of the functions $\Phi$ and $\rho$, we note that by estimates (43) and (44) the solution $B$ satisfies $\left(\eta, B(\eta, \tau), B_{\eta}(\eta, \tau)\right) \in \tilde{\mathcal{R}}$, with

$$
\tilde{\mathcal{R}}=\mathcal{R} \cap\{0 \leq v \leq M, 0 \leq-w \leq \bar{M}\},
$$

where $\mathcal{R}=\{\eta>0, v \geq 0, w \leq 0\} \cup\{\eta=0, v \geq 0, w=0\}$.

The functions $\rho$ and $\Phi$ are continuous in $\mathcal{R} \backslash\{\eta=\bar{\eta}, v>1\}$ with $\bar{\eta}>0$ defined later and they satisfy

$$
\frac{1}{C_{0}} \eta^{d+1} e^{-C_{0} \eta^{2}} \leq \rho(\eta, v, w) \leq \eta^{d+1} e^{-(d-2) \eta^{2} / 4 d} \quad \text { for } \quad(\eta, v, w) \in \tilde{\mathcal{R}},
$$

with $C_{0}=C_{0}(M)>0$ (see [24, Appendix]).

$$
|\Phi(\eta, v, w)| \leq C_{1} \eta^{d+1} e^{-(d-2) \eta^{2} / 4 d} \quad \text { for } \quad(\eta, v, w) \in \tilde{\mathcal{R}}
$$

for some positive constants $C_{1}(M)>0$ (see [24, Appendix]).

Proof of the claim. An integration over the interval $(a, b)$ of $(52)$ gives

$$
\int_{a}^{b} \int_{0}^{\ell(\tau)} \rho\left(\eta, B(\eta, \tau), B_{\eta}(\eta, \tau)\right) B_{\tau}^{2}(\eta, \tau) d \eta d \tau=E(a)-E(b)+\psi(a, b)
$$

where

$$
\begin{aligned}
\psi(a, b):= & \int_{a}^{b} \frac{1}{2} \ell(\tau) \Phi\left(\ell(\tau), B(\ell(\tau), \tau), B_{\eta}(\ell(\tau), \tau)\right) d \tau+ \\
& +\int_{a}^{b} B_{\tau}(\ell(\tau), \tau)\left[\int_{0}^{B_{\eta}(\ell(\tau), \tau)} \rho(\ell(\tau), B(\ell(\tau), \tau), s) d s\right] d \tau
\end{aligned}
$$

Since $B_{\tau}(\ell(\tau), \tau)=-B(\ell(\tau), \tau)-\frac{1}{2} \ell(\tau) B_{\eta}(\ell(\tau), \tau)$,

$$
B_{\tau}(\ell(\tau), \tau)=-T e^{-\tau}-\frac{1}{2} b_{r}\left(1, T\left(1-e^{\tau}\right)\right)
$$

Applying (39) at $r=1$ gives $\left|b_{r}\left(1, T\left(1-e^{\tau}\right)\right)\right| \leq d$ and consequently $B_{\tau}$ is uniformly bounded as $\tau \rightarrow \infty$. Employing this bound on $B_{\tau}$ and the estimates (54) and (55) we find

$$
\lim _{a \rightarrow \infty}\left\{\sup _{b>a} \psi(a, b)\right\}=0 .
$$

By (51), we have that there exists a sequence $n_{j} \rightarrow \infty$ such that $B^{n_{j}}(\eta, \tau)$ converges to $\bar{B}$ uniformly in compact subsets of $\left(\mathbb{R}^{+}\right)^{2}$. For any fixed $N$ we will prove for a subsequence satisfying $\lim _{j \rightarrow \infty}\left(n_{j+1}-n_{j}\right)=\infty$ that

$$
\lim _{n_{j} \rightarrow \infty} \int_{\mathcal{Q}_{N}} \rho\left(\eta, B^{n_{j}}(\eta, \tau), B_{\eta}^{n_{j}}(\eta, \tau)\right)\left(B_{\tau}^{n_{j}}\right)^{2}(\eta, \tau) d \eta d \tau=0
$$


where we recall that $\mathcal{Q}_{N}=\left\{(\eta, \tau): \mathbb{R}^{2}: 0 \leq \eta \leq N,|\tau| \leq N\right\}$. Since $\rho$ is bounded from below on bounded subsets of $\tilde{\mathcal{R}}$, it then follows that

$$
\int_{\mathcal{Q}_{N}} \bar{B}_{\tau}^{2} d \eta d \tau=\lim _{n_{j} \rightarrow \infty} \int_{\mathcal{Q}_{N}}\left(B_{\tau}^{n_{j}}\right)^{2}(\eta, \tau) d \eta d \tau=0,
$$

proving the claim. For all $j$ sufficiently large,

$$
N \leq\left(\chi_{d} \Theta T\right)^{-1 / 2} e^{\frac{1}{2}\left(n_{j}-N\right)} \quad \text { and } \quad n_{j+1}-n_{j} \geq 2 N .
$$

Consequently using (56), we find

$$
\begin{aligned}
& \int_{-N}^{N} \int_{0}^{N} \rho\left(\eta, B^{n_{j}}(\eta, \tau), B_{\eta}^{n_{j}}(\eta, \tau)\right)\left(B_{\tau}^{n_{j}}\right)^{2}(\eta, \tau) d \eta d \tau \\
& \leq \int_{-N}^{-N+n_{j+1}-n_{j}} \int_{0}^{\left(\chi \Theta^{*} T\right)^{-1 / 2} e^{\frac{1}{2}\left(n_{j}-N\right)}} \rho\left(\eta, B^{n_{j}}(\eta, \tau), B_{\eta}^{n_{j}}(\eta, \tau)\right)\left(B_{\tau}^{n_{j}}\right)^{2}(\eta, \tau) d \eta d \tau \\
& \leq \int_{n_{j}-N}^{n_{j+1}-N} \int_{0}^{\left(\chi \Theta^{*} T\right)^{-1 / 2} e^{\frac{1}{2\left(n_{j}-N\right)}} \rho\left(\eta, B(\eta, \tau), B_{\eta}(\eta, \tau)\right)\left(B_{\tau}\right)^{2}(\eta, \tau) d \eta d \tau} \\
& \leq E\left(n_{j}-N\right)-E\left(n_{j+1}-N\right)+\psi\left(n_{j}-N, n_{j+1}-N\right) .
\end{aligned}
$$

Hence applying (58), we discover

$$
\iint_{\mathcal{Q}_{N}} \rho\left(\eta, B^{n_{j}}(\eta, \tau), B_{\eta}^{n_{j}}(\eta, \tau)\right)\left(B_{\tau}^{n_{j}}\right)^{2}(\eta, \tau) d \eta d \tau \leq \limsup _{j \rightarrow \infty}\left[E\left(n_{j}-N\right)-E\left(n_{j+1}-N\right)\right] .
$$

Next we divide the expression $E\left(n_{j}-N\right)-E\left(n_{j+1}-N\right)$ into three integrals, choosing $K$ arbitrarily large:

$$
\begin{aligned}
& E\left(n_{j}-N\right)-E\left(n_{j+1}-N\right) \\
& =\int_{0}^{K}\left[\Phi\left(\eta, B^{n_{j}}(\eta,-N), B_{\eta}^{n_{j}}(\eta,-N)\right)-\Phi\left(\eta, B^{n_{j}}(\eta,-N), B_{\eta}^{n_{j}}(\eta,-N)\right] d \eta\right. \\
& +\int_{K}^{T^{-1 / 2} e^{\frac{n_{j}-N}{2}}} \Phi\left(\eta, B^{n_{j+1}}(\eta,-N), B_{\eta}^{n_{j+1}}(\eta,-N)\right) d \eta \\
& +\int_{K}^{T^{-1 / 2} e^{\frac{n_{j+1}-N}{2}}} \Phi\left(\eta, B^{n_{j}}(\eta,-N), B_{\eta}^{n_{j}}(\eta,-N)\right) d \eta .
\end{aligned}
$$

Integral (60) tends to zero as $j \rightarrow \infty$. In fact by the continuity of $\Phi$ in the second and third argument we obtain pointwise convergence and by the bounds (55) on $\Phi$, we apply the Dominated Convergence Theorem to conclude. Expressions (61) and (62) can be made arbitrarily small since they can be bounded by

$$
C \int_{K}^{\infty} \eta^{d+1} e^{-(d-2) \eta^{2} / 4 d} d \eta
$$

where $C$ is a positive constant, and $K$ can be chosen arbitrarily large. Thus we have proved (59), concluding the proof of the Theorem.

\section{Comparison results}

6.1. Comparison with the singular solution $\varphi_{S}$. This section closely follows [1]. From Section 4.2 , we recall that solutions $\varphi$ of (46)-(47) are classified by their intersections 
with $\varphi_{S}$. In this section we study the intersections of solutions $B$ of (29)-(31) with $\varphi_{S}$. Our results are closely related to the ones found in [1], where equation (33) was studied.

We first see that for $\Theta<1 /\left(2 d \chi_{d}\right)$ a solution $B$ of (29)-(31) intersects the singular solution $\varphi_{S}$ at least once in $\Pi(0)$ since

$$
\varphi_{S}(0)=\infty>B(0,0) \text {, and } \varphi_{S}\left(\left(\chi_{d} \Theta T\right)^{-1 / 2}\right)<B\left(\left(\chi_{d} \Theta T\right)^{-1 / 2}, 0\right)=T .
$$

On the other hand, for $\Theta \geq 1 /\left(2 d \chi_{d}\right)$ it can also be shown that $B$ intersects $\varphi_{S}$ at least once in $\Pi(0)$. Assuming the contrary, suppose that $B(\cdot, 0)<\varphi_{S}(\cdot)$ in $\Pi(0)$. By the maximum principle, we obtain $B<\varphi_{S}$ in $\Pi$. Therefore in the limit $\tau \rightarrow \infty$, thanks to Theorem 5.1 and since $B(0, \tau) \geq 1$ for all $\tau>0$, we find a solution $\varphi$ of (18) such that $\varphi<\varphi_{S}$. However we can show that every bounded nonzero solution $\varphi$ of (18) has to cross $\varphi_{S}$. This is equivalent to proving that there exists no solution $G$ of (49)-(50) such that $G(\eta)<2 d$ for $\eta \geq 0$. To check this, we assume that such a solution exists; we examine two cases. Suppose that for some $\eta^{*}$, we have $G_{\eta}\left(\eta^{*}\right)=0$ and $G\left(\eta^{*}\right)<2 d$. By (49), $G$ has a strict minimum at $\eta^{*}$, which contradicts the boundary condition (50). On the other hand if, $G(\eta)$ is increasing for all $\eta>0$, then for large $\eta$, equation (49) implies that $G_{\eta \eta}>0$, which also contradicts (50).

We conclude that there exists $\eta_{1} \in \Pi(0)$ such that $B\left(\eta_{1}, 0\right)=\varphi_{S}\left(\eta_{1}\right)$ and $B(\eta, 0)<$ $\varphi_{S}(\eta)$ for $\eta<\eta_{1}$.

LEMMA 6.1 ([24]). Under the assumptions (20) and (21), there exists a continuously differentiable function $\eta_{1}(\tau)$ with domain $[0, \infty)$ such that $\eta_{1}(0)=\eta_{1}$ and $B\left(\eta_{1}(\tau), \tau\right)=$ $\varphi_{S}\left(\eta_{1}(\tau)\right)$ for all $\tau \geq 0$.

Define the set $\Pi_{1}=\left\{(\eta, \tau) \mid 0<\eta<\eta_{1}(\tau)\right\}$ and the function

$$
\eta_{2}(\tau)=e^{\tau / 2} \cdot \sup \left\{\eta \in\left(\eta_{1},\left(\chi_{d} \Theta T\right)^{-1 / 2}\right]: H(s, 0) \geq 0 \text { for } s \in\left[\eta_{1}, \eta\right]\right\} .
$$

Since $H\left(\eta_{1}, 0\right)=0$ and $H_{\eta}\left(\eta_{1}, 0\right)>0$, the above supremum is finite. Define the set

$$
\Pi_{2}=\left\{(\eta, \tau) \mid \eta_{1}(\tau)<\eta<\eta_{2}(\tau)\right\}
$$

Let $F(\tau)=H\left(\eta_{2}(\tau), \tau\right)$. By definition of $\eta_{2}, F(0) \geq 0$. Also,

$$
\frac{d}{d \tau} F(\tau)=H_{\tau}\left(\eta_{2}(\tau), \tau\right)+\frac{1}{2} \eta_{2}(\tau) H_{\eta}\left(\eta_{2}(\tau), \tau\right)
$$

Using that $B_{\tau}+B+\eta B_{\eta} / 2>0$ in $\Pi$, we have $d\left[e^{\tau} F(\tau)\right] / d \tau \geq 0$. An integration yields $F(\tau) \geq 0$ for $\tau \geq 0$.

As was done in [1], applying the maximum principle, using Lemma 6.1, and noting that $H\left(\eta_{2}(\tau), \tau\right) \geq 0$ for $\tau \geq 0$, we can prove the following lemma and its corollary.

LEMma 6.2. The function $H(\eta, \tau)=B(\eta, \tau)-\varphi_{S}(\eta)$ satisfies $H<0$ in $\Pi_{1}$ and $H>0$ in $\Pi_{2}$.

Corollary 6.3. Assume the conditions in Lemma 6.1. For each $N>0$ there is $\tau_{N}>0$ such that for $\tau>\tau_{N}, B(\eta, \tau)$ intersect $\varphi_{S}(\eta)$ at most once in $\eta \in(0, N)$.

6.2. Intersection comparison. In this section we derive comparison results, which will be used to prove that $\omega$, the limit set (32), is a singleton. 
We start by considering the following linear equation with inhomogeneous boundary conditions:

$$
\begin{cases}v_{t}=v_{r r}+\frac{d+1}{r} v_{r}+a(r, t) v, & \text { for } 0<r<1, T_{1}<t<T_{2} \\ v_{r}(0, t)=0 & \text { for } T_{1}<t<T_{2} \\ v(1, t)=h(t) & \text { for } T_{1}<t<T_{2}\end{cases}
$$

where $T_{1}, T_{2}$ are positive constants and

$$
a \in L^{\infty}\left([0,1] \times\left(T_{1}, T_{2}\right)\right), \quad h \in C^{1}\left(\left(T_{1}, T_{2}\right)\right)
$$

are given functions. Moreover we assume

$$
h(t)>0 \text { for } T_{1}<t<T_{2} .
$$

To count the number of intersection of a solution $v$ of (63) with zero it is convenient to introduce the so-called zero number functional of (63), which is defined by

$$
z[v(\cdot, t)]=\#\{r \in[0,1]: v(r, t)=0\} .
$$

The following lemma provides properties of this zero number functional.

LEMma 6.4 ([32]). Let $v=v(r, t)$ be a nontrivial classical solution of (63) and assume that (64) and (65) hold. Then the following properties hold true:

1. $z[v(\cdot, t)]<\infty$ for any $T_{1}<t<T_{2}$;

2. $z[v(\cdot, t)]$ is monotone nonincreasing in time;

3. if $v\left(r_{0}, t_{0}\right)=v_{r}\left(r_{0}, t_{0}\right)=0$ for some $r_{0} \in[0,1]$ and $t_{0}>T_{1}$, then $z[v(\cdot, t)]$ drops strictly at $t=t_{0}$, that is, $z\left[v\left(\cdot, t_{1}\right)\right]>z\left[v\left(\cdot, t_{2}\right)\right]$ for any $T_{1}<t_{1}<t_{0}<t_{2}<T_{2}$.

From this lemma we deduce a property of intersection between a solution $\varphi$ of (18) and a solution $B$ of (29)-(31).

LEMma 6.5. Let $B$ be a bounded solution of (29)-(31) and let $\varphi$ be a solution of (4.2). Denote $Z(\tau)=\#\{r \in[0, \ell(\tau)]: B(\eta, \tau)=\varphi(\eta)\}$. Then the following properties hold true:

1. $Z(\tau)<\infty$ for any $\tau>\tau^{*}$

2. $Z(\tau)$ is monotone nonincreasing in time;

3. if $B\left(\eta_{0}, \tau_{0}\right)=\varphi\left(\eta_{0}\right)$ and $B_{\eta}\left(\eta_{0}, \tau_{0}\right)=\varphi_{\eta}\left(\eta_{0}\right)$ for $\tau_{0}>\tau_{1}$, and $\eta_{0} \leq \ell(\tau)$ then $Z\left(\tau_{1}\right)>Z\left(\tau_{2}\right)$ for any $\tau_{1}<\tau_{0}<\tau_{2}$.

Proof. Writing $\bar{V}=U-b$, where $U(r, t)=(T-t)^{-1} \varphi\left(r /\left(\chi_{d} \Theta(T-t)\right)^{1 / 2}\right)$, we have

$$
\begin{cases}\bar{V}_{t}=\bar{V}_{r r}+\frac{d+1}{r} \bar{V}_{r}+\frac{1}{d} r U \bar{V}_{r}+\left(\frac{1}{d} r b_{r}+b+U\right) \bar{V} & \text { for } 0<r<1,0<t<T, \\ \bar{V}_{r}(0, t)=0, \quad \bar{V}(1, t)=U(1, t)-b(1, t) & \text { for } 0<t<T .\end{cases}
$$

Let $T_{1}<T_{2}<T$. For the variable $V(r, t)=\exp \left(\int_{0}^{r} y U(y, t) d y /(2 d)\right) \bar{V}(r, t)$, we find

$$
\begin{cases}V_{t}=V_{r r}+\frac{d+1}{r} V_{r}+A(r, t) V & \text { for } 0<r<1, T_{1}<t<T_{2}, \\ V_{r}(0, t)=0, & \text { for } T_{1}<t<T_{2}, \\ V(1, t)=(U(1, t)-1) \exp \left(\frac{1}{2 d} \int_{0}^{1} y U(y, t) d y\right) & \text { for } T_{1}<t<T_{2},\end{cases}
$$

where

$$
A(r, t)=\frac{r}{d} b_{r}+b+U+\frac{1}{2 d} \int_{0}^{r} y U_{t}(y, t) d y-\frac{1}{4 d^{2}} r^{2} U^{2}-\frac{1}{2 d}\left(U+r U_{r}\right)-\frac{d+1}{2 d} U
$$


Note that $A \in L^{\infty}\left([0,1] \times\left(T_{1}, T_{2}\right)\right)$ since $b, b_{r}, U, U_{t}, U_{r} \in L^{\infty}\left([0,1] \times\left(T_{1}, T_{2}\right)\right)$. If we show that $V(1, t)$ does not change sign for $t>t_{0}$, then setting $T_{1}=t_{0}$ and using Lemma 6.4, we have proved the lemma.

We claim that there exists $\bar{t}_{0}$ such that $U_{t}(1, t)$ does not change sign for $t>\bar{t}_{0}$. By definition of $V(r, t)$, this claim implies that there exists $t_{0} \geq \bar{t}_{0}$ such that $V(1, t)$ does not change sign for $t>t_{0}$.

Since $U_{t}(r, t)=(T-t)^{-2}\left(\eta^{2} \varphi\right)_{\eta} /(2 \eta)$, if $r=1$ and $t>t^{*}$, then

$$
U_{t}(1, t)=(T-t)^{-2}\left(\eta^{2} \varphi\right)_{\eta} /(2 \eta) \quad \text { for } \quad t>t^{*}, \quad \eta>\eta^{*}\left(t^{*}\right)=\left(\chi_{d} \Theta\left(T-t^{*}\right)\right)^{-1 / 2} .
$$

From [10, Lemma A.1], we know that for a given $a \in(0,4 d)$, a solution $\varphi$ of $(46)$ satisfying

$$
\eta^{2} \varphi(\eta) \rightarrow a \text { as } \eta \rightarrow \infty
$$

is such that there exists $\bar{\eta}_{0}=\bar{\eta}_{0}(a)$ so that the sign of $\left(\eta^{2} \varphi\right)_{\eta}$ do not change on $\left[\bar{\eta}_{0}, \infty\right)$. Using (69), this implies that there exists $\bar{t}_{0}=\bar{t}_{0}\left(\bar{\eta}_{0}\right)$ such that the claim holds.

In general, for any $a \geq 0$, we know that, a solution $\varphi$ of (46) satisfying (70) admits the expansion

$$
\varphi(\eta)=a \eta^{-2}\left(1+b \eta^{-2}-c b \eta^{-4}\right)+O\left(\eta^{-6}\right) \text { as } \eta \rightarrow \infty
$$

where $b=\frac{(d-2)}{d}(a-2 d)$, and $c=(2 d-8)-\frac{d-3}{d} a$, see [28, (2.12)]. This can be done rigorously, by adapting the proofs found in [32] describing the asymptotic behaviour of bounded self-similar solutions of (33).

Using (71) and (69), we find that the sign of $U_{t}$ for $t$ near $T$ depends on the value of $\operatorname{sign}(a-2 d)$. If $a>2 d$, then there exists $t_{0}^{+}$such that $U_{t}$ is positive for $t>t_{0}^{+}$and if $a<2 d$, then there exists $t_{0}^{-}$such that $U_{t}$ is negative for $t>t_{0}^{-}$. This proves the claim and consequently the lemma.

7. Proofs of main results. We start by proving that the $\omega$-limit set of problem (29)(31) is a singleton.

TheOREM 7.1. Assume the hypotheses of Theorem 2.3. Then the set $\omega$ defined in (32) is a singleton.

Proof. For this proof we extend a solution $B$ of $(29)-(31)$ to all $\left(\mathbb{R}^{+}\right)^{2}$ by setting $B(\eta, \tau)=$ $e^{-\tau} T$ for $(\eta, \tau) \in\left(\mathbb{R}^{+}\right)^{2} \backslash \Pi$. We also define the weight function $\rho^{*}(\eta)=e^{-\eta^{2} / 4}$ for $\eta>0$.

The hypothesis (25) implies that $B$ is uniformly bounded; Theorem 5.1 therefore states that $\omega$ is non-empty, and that each $\varphi \in \omega$ is a solution of (46)-(47).

We claim that for each $\varphi \in \omega$ there exists $\tau^{*}>0$ such that $B(0, \tau)-\varphi(0)$ never changes sign in $\left[\tau^{*}, \infty\right)$. By contradiction, we assume that there exists a sequence $\tau_{k}$, such that $\tau_{k} \rightarrow \infty$, and $B\left(0, \tau_{k}\right)=\varphi(0)$. Since $B_{\eta}\left(0, \tau_{k}\right)=\varphi_{\eta}(0)=0$, by Lemma 6.5 the function $Z(\tau)$ has to decrease at least by one. However this cannot happen an infinite number of times. This proves the claim.

Suppose now that $\omega$ is not a singleton. Since the $\omega$-limit set is connected, closed, and nonempty, it contains an infinite number of elements. We select three different elements $\varphi_{1}, \varphi_{2}, \varphi_{3}$ in the $\omega$-limit set. Since these functions are different and each solves (18), we may assume that $\varphi_{1}(0)<\varphi_{2}(0)<\varphi_{3}(0)$. By the claim above, $B(0, \tau)-\varphi_{2}(0)$ never 
changes sign in $\left[\tau^{*}, \infty\right)$. This contradicts the fact that $\varphi_{1}$ and $\varphi_{3}$ are elements of $\omega$; it follows that $\omega$ must be a singleton.

We now conclude the proof of Theorems 2.3 and 2.1, and Corollary 2.2.

Proof of Theorem 2.3. By the previous theorem $\omega$ is a singleton, say $\{\bar{B}\}$. From Corollary 6.3 , we find that for every $N>0$ there exists a $\tau_{N}>0$ such that the solution $B(\eta, \tau)$ intersects $\varphi_{S}(\eta)$ at most once in $\eta \in[0, N]$ for each $\tau>\tau_{N}$. This implies that in the limit $\tau \rightarrow \infty, \bar{B}$ intersects $\varphi_{S}$ at most once, concluding the proof.

Proof of Theorem 2.1. Since $b$ and $U_{1}(r, t)=(T-t)^{-1} \varphi_{1}\left(r /\left(\chi_{d} \Theta(T-t)\right)^{1 / 2}\right)$ are solutions of (14) with the same blow up time, $\bar{V}=b-U_{1}$ satisfies equation (67). Using that $U_{1}(r, t)=2 d /\left((d-2)(T-t)+r^{2} /\left(2 \chi_{d} \Theta\right)\right)$, we find

$$
\bar{V}(1, t)=\left(1-U_{1}(1, t)\right)>0 \quad \text { if } \quad \Theta \leq 1 /\left(4 d \chi_{d}\right) \text { for any } t<T \text {. }
$$

The functions $U_{1}$ with $b$ necessarily intersect exactly once for all $t$, since non-intersection implies that the solutions must have different times of blow-up [34, p. 271]. It follows that $b(0,0)<U_{1}(0)$, and one finds $(T-t) b(0, t) \leq 2 d /(d-2)$. An application of Theorem 2.3 proves the theorem.

Proof of Corollary 2.2. If $b_{0} \equiv 1$ and $\Theta<1 /\left(2(d+2) \chi_{d}\right)$, we know from [7, Theorem 2] that the corresponding solution $b$ blows up. Now assuming $\Theta \leq 1 /\left(4 d \chi_{d}\right)<1 /(2(d+$ 2) $\chi_{d}$ ), we can apply Theorem 2.1 to conclude.

8. Linear stability of blow-up profiles. In this section, we study the linear stability of the blow-up profiles $\varphi_{1}$ and $\varphi^{*}$ defined in (19).

Let $B$ be a solution of (29)-(31) and let $\varphi$ be a solution of (18). The idea is to study the linearized equation for the difference $\Phi(\eta, \tau):=B(\eta, \tau)-\varphi(\eta)$, i.e.

$$
\Phi_{\tau}=\Phi_{\eta \eta}+\frac{d+1}{\eta} \Phi_{\eta}+\left(\frac{1}{d} \varphi-\frac{1}{2}\right) \eta \Phi_{\eta}+\left(\frac{1}{d} \eta \varphi_{\eta}+2 \varphi-1\right) \Phi .
$$

Here, we are implicitly assuming that sufficiently close to blow-up only the linear terms play a role in describing the singularity formation.

For the stability analysis, we consider, for a given $\lambda>0$, a solution of (72) of the form $\psi_{\lambda}(\eta) e^{\lambda \tau}$. Then $\psi_{\lambda}(\eta)$ satisfies

$$
\left(\psi_{\lambda}\right)_{\eta \eta}+\frac{d+1}{\eta}\left(\psi_{\lambda}\right)_{\eta}+\left(\frac{1}{d} \varphi-\frac{1}{2}\right) \eta\left(\psi_{\lambda}\right)_{\eta}+\left(\frac{1}{d} \eta \varphi_{\eta}+2 \varphi-1-\lambda\right) \psi_{\lambda} .
$$

First we consider the stability of $\varphi=\varphi_{1}$. Concerning boundary conditions, we note that at $\eta=0$, we find either $\psi_{\lambda} \sim 1$ or $\psi_{\lambda} \sim 1 / \eta^{d}$. To have $\psi_{\lambda}$ bounded near 0 , we impose

$$
\left(\psi_{\lambda}\right)_{\eta}(\eta) \rightarrow 0, \quad \text { as } \quad \eta \rightarrow 0 \text {. }
$$

For large $\eta$, we can either have a) $\psi_{\lambda} \sim \eta^{-(2 \lambda+3)} e^{\eta^{2} / 4}$ or b) $\psi_{\lambda} \sim \eta^{2 \lambda-2}$. We see that both behaviours diverge with $\eta$, however the asymptotic b) in terms of $r$ and $t$ is bounded as $t \rightarrow T$. Consequently to obtain the power-like behaviour b) at infinity, we prescribe

$$
\psi_{\lambda}(\eta) e^{-\eta} \rightarrow 0, \quad \text { as } \quad \eta \rightarrow \infty .
$$

Now solving equation (73) together with (74) and (75), we find a sequence of solutions of (72) given by $\left\{e^{\lambda_{n} \tau} \psi_{n}(\eta)\right\}_{n \in \mathbb{N} \cup\{0\}}$, with $\lambda_{0}>\lambda_{1}>\ldots$, where $\psi_{n}:=\psi_{\lambda_{n}}$. If the blow-up 
time $T>0$ is chosen correctly in the definition of $\eta$ and $\tau$, we can eliminate, see [11], the first mode $(n=0)$ corresponding to change of blow-up and write

$$
B(\eta, \tau)=\varphi(\eta)+\psi_{1}(\eta) e^{\lambda_{1} \tau}+O\left(e^{\lambda_{2} \tau}\right)
$$

Therefore, from the sign of $\lambda_{1}$ we obtain the linear stability of $\varphi$.

In [11], Brenner et al. proved, using (72), the following stability result for various blow-up profiles.

THEOREM 8.1. Every solution $\varphi$ of (46) satisfying $\eta \varphi_{\eta} / \varphi \rightarrow 2$ as $\eta \rightarrow \infty$ has an unstable mode corresponding to changing the blow-up time. Also, a blow-up profile with $k$ intersects with the singular solution $\varphi_{S}$ has at least $k-1$ additional unstable modes.

In addition, the authors in [11] found numerically that $\lambda_{1}<0$ when $\varphi=\varphi_{1}$ and $d>2$. In particular, they found $\lambda_{1}=-0.272 \ldots$ when $d=3$. This implies that $\varphi_{1}$ is linearly stable for all $d>2$.

For $\varphi=\varphi^{*}$, we can proceed as above and solve the eigenvalue problem for (72). Using (73) with $\varphi=\varphi^{*}$, we find that $\left\langle\psi_{\lambda}, \lambda\right\rangle$ satisfies

$$
\left(\psi_{\lambda}\right)_{\eta \eta}+\left(\frac{d+1}{\eta}-\frac{d-2}{2 d} \eta\right)\left(\psi_{\lambda}\right)_{\eta}+(1-\lambda) \psi_{\lambda}=0
$$

with (74) and (75). These boundary conditions are chosen by the same arguments as for $\varphi=\varphi_{1}$, in this case however we have either $\psi_{\lambda} \sim \eta^{\frac{2 d}{d-2}(\lambda-1)-d-2} e^{\frac{(d-2)}{4 d} \eta^{2}}$ or $\psi_{\lambda} \sim$ $\eta^{\frac{2 d}{d-2}(1-\lambda)}$ as $\eta \rightarrow \infty$. Note that changing $\eta$ by $(-\eta)$ equation (76) remains invariant, so only solutions consisting of even powers are allowed. Therefore the only possible solutions are a sequence of the form

$$
\psi_{n}(\eta)=\sum_{i=0}^{n} A_{i} \eta^{2 i} \quad \text { for any } \quad n=0,1,2,3 \ldots,
$$

where the coefficients are given by $A_{i}(2 i(2 i-1)+(d+1) 2 i)=A_{i-1}(1-\lambda-2 i(d-2) / 2 d)$ for $i=1,2, \ldots$ and $A_{0}$ an arbitrary constant. This means that when $(1-\lambda-2(n+1)(d-$ $2) / 2 d)=0$, we find an explicit polynomial solution of degree $2 n$ with a respective $\lambda$ given by

$$
\lambda_{n}=\frac{d-n(d-2)}{d} .
$$

Consequently, we have obtained an explicit sequence of solutions $\left\{\left\langle\psi_{n}, \lambda_{n}\right\rangle\right\}_{n \in \mathbb{N} \cup\{0\}}$ for the eigenvalue problem (76). The eigenvalue $\lambda_{0}=1$ corresponds to the unstable mode of change of blow-up time. The formula of $\lambda_{n}$ says that $\varphi^{*}$ is linearly unstable, since $\lambda_{1}>0$ for all $d>2$.

\section{Related models}

9.1. Isothermal model for $d=2$. In this section we revisit some known results of blow-up for the isothermal model when $d=2$, see [26] for details. This case is critical for blow-up, since for $d<2$ blow-up does not occur, see [11]. It is known [28] that no bounded (self-similar) solution of (46) satisfying $\varphi(\eta) \rightarrow 0$ as $\eta \rightarrow \infty$ exists. From this result, we expect a different blow-up behaviour in comparison with the case $d>2$. In fact, it was shown in [26] that there exists a solution $B$ of (29)-(31) that converges to an element of 
$F_{S}=\left\{\varphi(\eta)=K / \eta^{2} \mid K>0\right\}$. Note that $F_{S}$ is a family of singular stationary solutions of equation (46). This implies immediately that

$$
B(0, \tau)=(T-t) b(0, t) \rightarrow \infty \quad \text { as } \quad t \rightarrow T(\tau \rightarrow \infty) .
$$

This clearly differs from what we expect for $d>2$. To find $K>0$, i.e. an element $\varphi$ of $F_{S}$, such that the convergence holds, we define $W(\eta, \tau)=\int_{0}^{\eta} B(y, \tau) y^{3} d y$, which satisfies

$$
W_{\tau}=W_{\eta \eta}-3 \frac{W_{\eta}}{\eta}-\frac{\eta}{2} W_{\eta}-W+\frac{1}{4}\left(\frac{W_{\eta}}{\eta}\right)^{2} .
$$

This formally suggests that $W(\eta, \tau) \rightarrow 4 \eta^{2}$ as $\tau \rightarrow \infty$, implying that $K=8$, that is, $B(\eta, \tau) \rightarrow 8 / \eta^{2}$ as $\tau \rightarrow \infty$. Since $B_{\eta}(0, \tau)=0$, the convergence to a singular solution gives rise to a boundary layer near the origin. To quantify this effect, we make the change of variables $\Phi(\xi, \tau)=\eta^{2} B(\eta, \tau)$ with $\xi=\eta / \epsilon(\tau)$ where $\lim _{\tau \rightarrow \infty} \epsilon(\tau)=0$. In [26], using this transformation, it was proved that

$$
B(\eta, \tau)\left(1+\frac{\epsilon(\tau)^{2}}{\eta^{2}}\right) \sim \frac{8}{\eta^{2}} \quad \text { as } \quad \tau \rightarrow \infty
$$

uniformly on sets $\eta \leq \epsilon(\tau)$ where $\epsilon(\tau)=\tilde{K} \exp \left(-\sqrt{2} \tau^{1 / 2} / 2\right)(1+o(1))$ as $\tau \rightarrow \infty$ and where $\tilde{K}$ is a positive constant depending on $\Theta$. The asymptotic expression for $\epsilon(\tau)$ is found by studying the expansion of a solution $W(\eta, \tau)$ of $(77)$ about $\bar{W}(\eta)=4 \eta^{2}$. From (78), we get

$$
(T-t) b(0, t) \tilde{K} \exp \left(\sqrt{2}|\log (T-t)|^{1 / 2}\right) \rightarrow 8, \quad \text { as } \quad t \rightarrow T .
$$

These results mean that the corresponding $n(r, t)$ converges to a delta function at the origin with mass $8 \pi \Theta$.

The stability of this blow-up behavior has been proved analytically in [37] and numerically in [13].

This problem has special importance for bacteria aggregation, where a two-dimensional approximation can be assumed. We can find aggregation in a so-called quasi-two-dimensional geometry (e.g., Dictyostelium discoideum, see [29], [30], and [37]), and also find that bacteria form cylindrical aggregates (e.g., Escherichia Coli, see [2] and [9]).

9.2. Energy conservation model. In astrophysics, a model was proposed by Chavanis et al. [15] considering energy conservation for the system of particles. To model that, they introduce a variable temperature $\Theta=\Theta(t)>0$ and replace (1) by

$$
n_{t}=\operatorname{div}(\Theta(t) \nabla n+n \nabla \phi) .
$$

They complement this equation with conservation of total energy $E \in \mathbb{R}$,

$$
E=\kappa \Theta(t)+\int_{\Omega} n(x, t) \phi(x, t) d x .
$$

This energy is the sum of kinetic energy with specific heat $\kappa>0$ and potential energy. Well posedness for the system requires $\Theta(0)>0$ and $n_{0}$ to be as for the isothermal case, see [17]. In this model blow-up occurs for $d=3$, but not for $d=2$, see [8]. In fact for $d=3$, blow-up occurs for bounded initial data, $E<1 / 4 \pi$ and $\kappa>6$, [17]. Assuming 

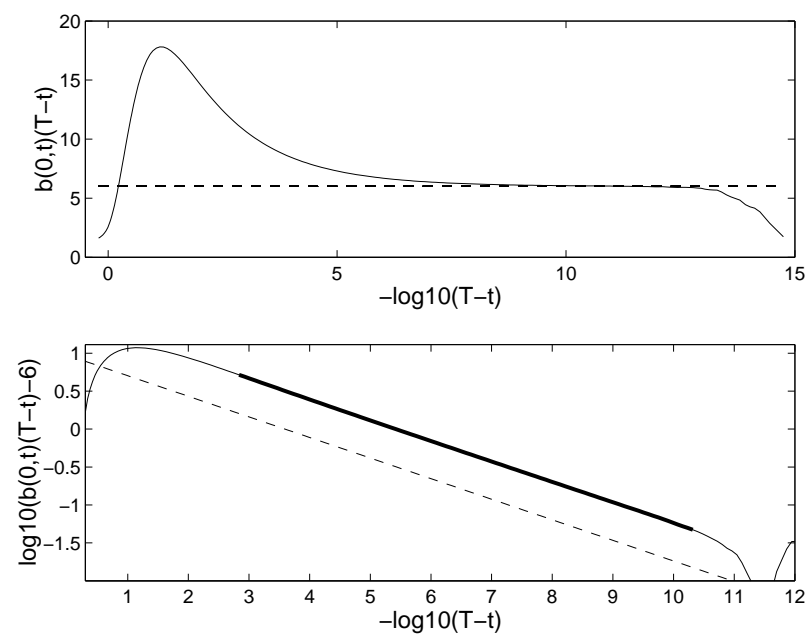

Fig. 2. Case $E=0.3 /(12 \pi), \kappa=d=3$. The upper figure shows that $B(0, \tau) \rightarrow 6$ as $\tau \rightarrow \infty$, $\bar{B}=6$ is the dotted line. The bottom figure shows the rate of convergence. The dotted line has a slope fitting the bold line.
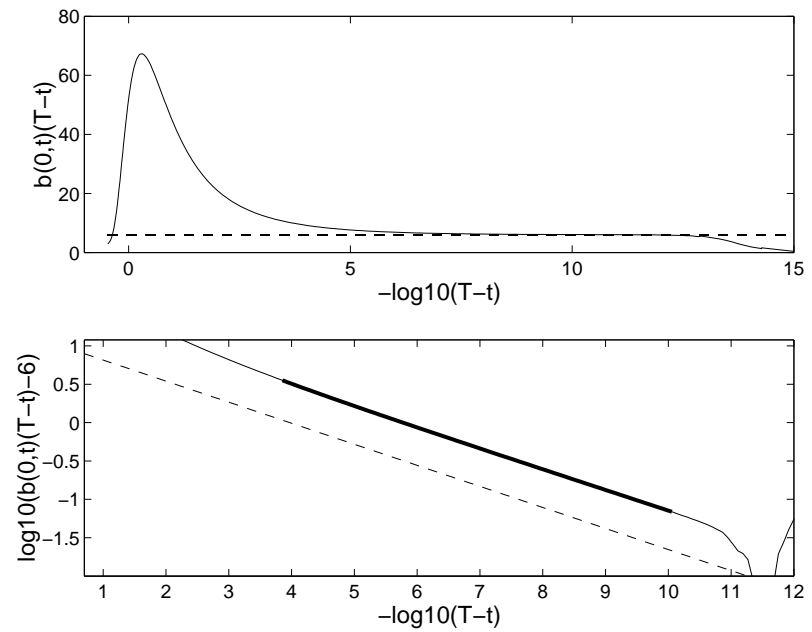

Fig. 3. Case $E=0.846 /(12 \pi), \kappa=d=3$. The upper figure shows that $B(0, \tau) \rightarrow 6$ as $\tau \rightarrow \infty$, $\bar{B}=6$ is the dotted line. The bottom figure shows the rate of convergence. The dotted line has a slope fitting the bold line.

radial symmetry, the integral relation in terms of $b(r, t)$ becomes

$$
E=\kappa \Theta(t)-\frac{1}{d \chi_{d}} \int_{0}^{1} b^{2}(r, t) r^{d+1} d x \text { for } t \geq 0 .
$$

We conjecture that for a generic class of initial data, blow-up for this model is essentially equivalent to the self-similar blow-up for the isothermal model. We present here numerical computations to corroborate that, see other simulations in [23, Chapter 4]. We take $d=3, \kappa=3$ and the cases $E=0.846 /(12 \pi)$ and $E=0.3 /(12 \pi)$ (this corresponds 


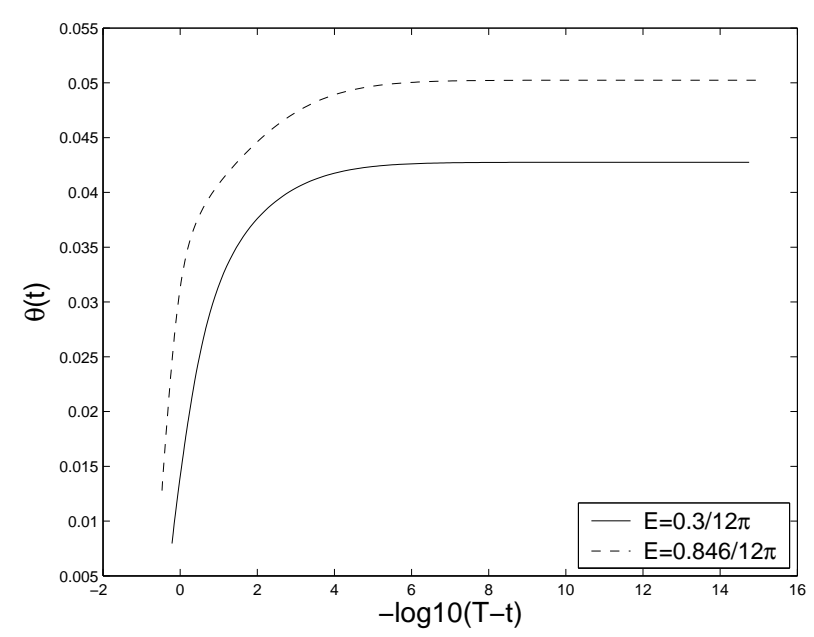

Fig. 4. The evolution of temperature for two different energies and $\kappa=d=3$. Clearly the temperature converges to a constant.
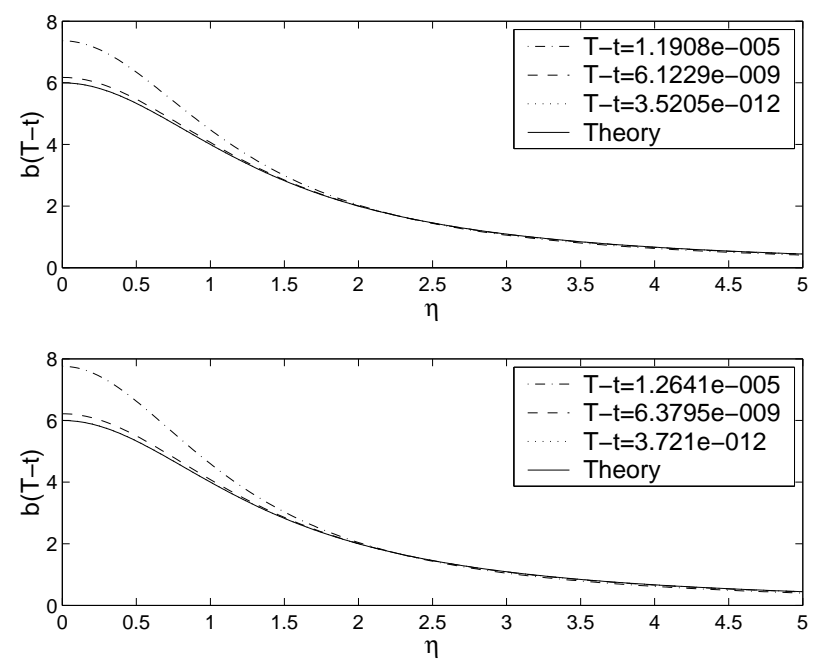

Fig. 5. The evolution of the density profiles for two energies $(E=0.3 /(12 \pi)$ in the upper figure and $E=0.846 /(12 \pi)$ in the bottom figure) with $\kappa=d=3$. It shows convergence to the blow-up profile $\varphi_{1}(\eta)$ (Theory).

to $E R / G M^{2}=-0.359$ and $E R / G M^{2}=-0.45$ in the notation of [14], [16], and [35]). Figures 2 and 3 show that

$$
(T-t) b(0, t) \rightarrow 6 \quad \text { as } \quad t \rightarrow T
$$

with a particular rate of convergence. This rate is very close to the theoretical value predicted by the linear stability analysis for the isothermal case, that is, $\lambda=-0.272 \ldots$. In fact the dotted lines fit slopes $\lambda=-0.27134 \ldots$ in Figure 2 and $\lambda=-0.2744$ in 
Figure 3. Figure 4 shows that $\Theta(t)$ converges to a constant approaching blow-up. Finally in Figure 5, we observe the convergence of solutions to the blow-up profile $\varphi_{1}(\eta)$.

The numerical method uses a moving mesh, which evolves following carefully chosen PDE, called MMPDE. Problem (14)-(16), and (79) with variable $\Theta$, is coupled with this MMPDE and the system is solved using a collocation method. This approach allow us to reach large values of densities and compute times closer to blow-up. The results obtained are reliable until $T-t \sim 10^{-10}$ approximately. For details we refer to [23, Chapter 4] and the reference therein.

9.3. Momentum conservation model. A classical problem in astrophysics is to consider that momentum is conserved for the isothermal model. To allow this property, we introduce a velocity $\mathbf{v}=\mathbf{v}(x, t)$ defined for each point in space and time. We refer to [12] and the references therein for details. Let $B\left(0, R_{c}\right)$ be a ball in $\mathbb{R}^{3}$ of radius $R_{c}>0$ and consider in this domain the problem

$$
\begin{array}{r}
n_{t}+\operatorname{div}(n \mathbf{v})=0 \\
\mathbf{v}_{t}+\mathbf{v} \cdot \nabla \mathbf{v}=-\frac{1}{n} \nabla p-4 \pi G \nabla \phi \\
\Delta \phi=n
\end{array}
$$

where $G$ is the gravitational constant and $p=p(x, t)$ is the pressure. Here we prescribe no-flux and zero velocity and potential at the boundary. We start with $n_{0}(x)=\rho_{0}$ constant. We consider in the following $p=c^{2} n$, the isothermal equation of state, where $c$ is the speed of sound in the initial uniform quiescent interstellar gas. Assuming radial symmetry and taking $u=u(r, t)=\mathbf{v}(x, t)$ with $r=|x|$, equations (80)-(82) for $\langle n=$ $n(r, t), \phi=\phi(r, t), u=u(r, t)\rangle$, become

$$
\begin{array}{r}
n_{t}+\frac{1}{r^{2}}\left(r^{2} n u\right)_{r}=0, \\
u_{t}+u u_{r}=-\frac{n_{r}}{n}-N \phi_{r}, \\
\frac{1}{r^{2}}\left(r^{2} \phi_{r}\right)_{r}=n .
\end{array}
$$

Here $N=4 \pi \rho_{0} R^{2} G / c^{2}$ is the dimensionless constant, which determines the dynamics. The stationary solutions of this system coincide with the stationary solutions of the isothermal model. Now however the dynamics is completely different, we have a hyperbolic system where $1 / N$ can be related to $\Theta$ in the isothermal model. In the following, the analysis is shown only for the variable $n(r, t)$, since we are interested in a comparison with the constant temperature model. Using the hyperbolicity of this problem we can show that there exist functions $\bar{n}=\bar{n}(t)$ and $\bar{r}=\bar{r}(t)$ such that we have a solution of the form

$$
n(r, t)=\bar{n}(t) \quad \text { for } \quad r \leq \bar{r}(t)
$$

for $t \leq \bar{t}_{f}$ where $\bar{t}_{f}$ is defined by $\bar{r}\left(\bar{t}_{f}\right)=0$. Clearly, $\bar{n}$ and $\bar{r}$ depend on $N$. For $N<$ $N_{C}=3 \pi^{2} / 2=14.8 \ldots$, we have that the final core density will be finite, $\bar{n}\left(\bar{t}_{f}\right)<\infty$. For $N>N_{C}$ the value of $\bar{n}\left(\bar{t}_{f}\right)$ is infinite. 
There exist stationary solutions of the system when $N<N_{E}=7.5$. However, there exists a region $N_{M}=6.6<N<N_{E}$ where collapse still occurs, this suggest that the equilibrium solution (which is linearly stable) is not a global atractor, and the initial state is far from equilibrium.

For $N_{M}<N<N_{C}$ the time of blow-up, $T>0$, is estimated from below by $\bar{t}_{f}(T>$ $\left.\bar{t}_{f}\right)$. The dynamics for $t \in\left(0, \bar{t}_{f}\right]$ is given by the solution (86) and for $t>\bar{t}_{f}$ is determined by self-similar solutions (Larson-Penston), a one-parameter family of solutions, which in terms of $n(r, t)$ has blow-up of the form

$$
n_{*}(r, t)=(T-t)^{-2} R\left(\frac{r}{T-t}\right)
$$

where

$$
R(\eta) \sim R_{0}-\frac{R_{0}\left(3 R_{0}-2\right)}{18} \eta^{2} \quad \text { at } \quad \eta=0, \quad \text { and } \quad R(\eta)=O\left(\eta^{-2}\right) \quad \text { as } \quad \eta \rightarrow \infty,
$$

and $R_{0}>0$ is the scaled self-similar density at the origin. For $N>N_{C}, \bar{t}_{f}$ is a good estimate for $T$. In this case the solution of the form (86) is unstable. Therefore depending on the initial disturbances, we can have Larson-Penston similarity or other type of density profile, which could even be nonmonotone.

\section{References}

[1] J. Bebernes and D. Eberly, A description of self-similar blow-up for dimension $N \geq 3$, Ann. Inst. H. Poincaré, Anal. Nonlin. 5 (1988), 1-22.

[2] M. D. Betterton and M. P. Brenner, Collapsing bacterial cylinders, Phys. Rev. E 64061904 (2001).

[3] P. Biler, Existence and nonexistence of solutions for a model of gravitational interaction of particles, III, Colloq. Math. 68 (1995), 229-239.

[4] P. Biler, D. Hilhorst, and T. Nadzieja, Existence and nonexistence of solutions for a model of gravitational interaction of particles, II, Colloq. Math. 67 (1994), 297-308.

[5] P. Biler, A. Krzywicki, and T. Nadzieja, Self-interaction of Brownian particles coupled with thermodynamic processes, Rep. Math. Phys. 42 (1998), 359-372.

[6] P. Biler and T. Nadzieja, Existence and nonexistence of solutions for a model of gravitational interaction of particles, I, Colloq. Math. 66 (1994), 319-334.

[7] P. Biler and T. Nadzieja, Growth and accretion of mass in an astrophysical model II, Applicationes Math. 23 (1995), 351-361.

[8] P. Biler and T. Nadzieja, Global and exploding solutions in a model of self-gravitating systems, Rep. Math. Phys. 52 (2003), 205-225.

[9] M. P. Brenner, L. S. Levitov, and E. O. Budrene, Physical mechanisms for chemotactic pattern formation by bacteria, Biophysical J. 74 (1998), 1677-1693.

[10] M. P. Brenner, P. Constantin, L. P. Kadanoff, A. Schenkel, and S. C. Venkataramani, Blowup in the chemotaxis equation, Technical Report (1998).

[11] M. P. Brenner, P. Constantin, L. P. Kadanoff, A. Schenkel, and S. C. Venkataramani, Diffusion, attraction, and collapse, Nonlinearity 12 (1999), 1071-1098.

[12] M. P. Brenner and T. P. Witelski, On spherically symmetric gravitational collapse, J. Stat. Physics 93 (1998), 863-869. 
[13] C. J. Budd, R. Carretero-González and R. D. Russell, Precise computations of chemotactic collapse using moving mesh methods, submitted to J. Comput. Physics (2003).

[14] P. H. Chavanis, C. Rosier, and C. Sire, Thermodynamics of self-gravitating system, Phys. Rev. E 66, 036105 (2002).

[15] P. H. Chavanis, C. Sire, and R. Robert, Statistical mechanics of two-dimensional vortices and collisionless stellar systems, Astrophys. J. 471 (1996), 385-399.

[16] P. H. Chavanis and C. Sire, Anomalous diffusion and collapse of self-gravitating Langevin particles in D dimensions, Phys. Rev. E 69 (2004), 016116.

[17] C. J. van Duijn, I. A. Guerra, and M. A. Peletier, Global existence conditions for a nonlocal problem arising in statistical mechanics, Adv. Diff. Equations 9 (2004), 133-158.

[18] M. Escobedo, M. A. Herrero, and J. J. L. Velázquez, A nonlinear Fokker-Planck equation modelling the approach to thermal equilibrium in a homogeneous plasma, Trans. Amer. Math. Soc. 350 (1998), 3837-3901.

[19] A. Friedman and B. McLeod, Blow-up of positive solutions of semilinear heat equations, Indiana Univ. Math. J. 34 (1985), 425-447.

[20] V. A. Galaktionov, On asymptotic self-similar behaviour for a quasilinear heat equation: single point blow up, SIAM J. Math. Anal. 26 (1995), 675-693.

[21] Y. Giga and R. Kohn, Asymptotically self-similar blowup of semilinear heat equations, Comm. Pure Appl. Math. 38 (1985), 883-901.

[22] Y. Giga and R. Kohn, Characterizing blowup using similarity variables, Indiana Univ. Math. J. 36 (1987), 1-40.

[23] I. A. Guerra, Stabilization and blow-up for some multidimensional nonlinear PDE's, $\mathrm{PhD}$ Thesis, Technical University Eindhoven TU/e, 2003.

[24] I. Guerra and M. Peletier, Asymptotic self-similar blow-up for a diffusion-atraction problem, to be submitted, (2004).

[25] A. Haraux, Systèmes Dynamiques Dissipatifs et Applications, Recherches en Mathématiques Appliquées, RMA 17, Masson, 1991.

[26] M. A. Herrero and J. L. L. Velázquez, Singularity patterns in a chemotaxis model, Math. Ann. 306 (1996), 583-623.

[27] M. A. Herrero, E. Medina, and J. L. L. Velázquez, Finite-time aggregation into a single point in a reaction-diffusion system, Nonlinearity, 10 (1997), 1739-1754.

[28] M. A. Herrero, E. Medina, and J. L. L. Velázquez, Self-similar blow-up for a reactiondiffusion system, J. Comput. Appl. Math. 97 (1998), 99-119.

[29] W. Jäger and S. Luckhaus, On explosions of solutions to a system of partial differential equations modelling chemotaxis, Trans. Amer. Math. Soc. 329 (1992), 819-824.

[30] E. F. Keller and L. A. Segel, J. Theor. Biol. 26 (1970), 399-415,

[31] H. Matano, Convergence of solutions of one-dimensional parabolic equations, J. Math. Kyoto Univ. 18 (1978), 221-227.

[32] J. Matos, Convergence of blow-up solutions of nonlinear heat equations in the supercritical case, Proc. Roy. Soc. Edinburgh Sect. A 129 (1999), 1197-1227.

[33] J. Matos, Self-similar blow up patterns in supercritical semilinear heat equations, Commun. Appl. Anal. 5 (2001), 455-283.

[34] A. A. Samarskii, V. A. Galaktionov, S. P. Kurdyumov, and A. P. Mikhailov, Blow-up in Problems for Quasilinear Parabolic Equations, Walter de Gruyter, Berlin, 1995.

[35] C. Sire and P. H. Chavanis, Thermodynamics and collapse of self-gravitating Brownian particles in D dimensions, Phys. Rev. E, 66 (2002), 046133. 
[36] J. J. L. Velázquez, Classification of singularities for blowing up solutions in higher dimensions, Trans. Amer. Math. Soc. 338 (1993), 441-464.

[37] J. J. L. Velázquez, Stability of some mechanisms of chemotactic aggregation, SIAM J. Appl. Math. 62 (2002), 1581-1633.

[38] G. Wolansky, On steady distributions of self-attracting clusters under friction and fluctuations, Arch. Rational Mech. Anal. 119 (1992), 355-391.

[39] T. I. Zelenyak, Stabilization of solutions of boundary value problems for a second order parabolic equation with one space variable, Differ. Equat. 4 (1968), 17-22. 